\title{
INVARIANTS OF FINITE REFLEGTION GROUPS
}

\author{
ROBERT STEINBERG
}

Let us define a reflection to be a unitary transformation, other than the identity, which leaves fixed, pointwise, a (reflecting) hyperplane, that is, a subspace of deficiency 1 , and a reflection group to be a group generated by reflections. Chevalley (1) (and also Coxeter (2) together with Shephard and Todd (4)) has shown that a reflection group $G$, acting on a space of $n$ dimensions, possesses a set of $n$ algebraically independent (polynomial) invariants which form a polynomial basis for the set of all invariants of $G$. Our aim here is to prove:

Theorem. Let $G$ be a finite reflection group, acting on a space $V$ of finite dimension. Let $J$ be the Jacobian (matrix) of a basic set of invariants of $G$, computed relative to any basis of $V$. Let $p$ be any point of $V$. Then the following numbers are equal:

(a) the maximum number of linearly independent reflecting hyperplanes containing $p$;

(b) the maximum rank of $1-x$ for all $x$ in $G$ for which $x p=p$;

(c) the nullity of $J$ at $p$.

The equality of the numbers defined in (b) and (c) is the essence of a conjecture of Shephard (3).

Throughout the paper, $G$ is a reflection group, of finite order $g$, acting on a space $V$ of $n$ dimensions. The symbols $L_{1}, \ldots, L_{v}$ denote the hyperplanes in which reflections of $G$ take place, as well as non-zero linear forms which vanish on the corresponding hyperplanes, and for each $i, a_{i}$ is a corresponding nonzero normal vector, $r_{i}$ is the order of the (cyclic) subgroup of $G$ which leaves $L_{i}$ fixed pointwise, and $R_{i}$ is a generator of this subgroup. Finally, $I_{1}, \ldots, I_{n}$ are basic invariants of $G ; d_{1}, \ldots, d_{n}$ are their degrees; and $J$ generically denotes their Jacobian, relative to whatever basis is at hand.

Lemma. For some non-zero scalar $c$,

$$
\operatorname{det} J=c \prod_{i=1}^{v} L_{i}^{r_{i}-1} \text {. }
$$

A proof of this well-known result will be included because it and the corollary below play a key role in the proof of the theorem. Choose an orthonormal basis of $V$ so that the first co-ordinate $x_{1}$ is a multiple of $L_{1}$. If $I$ is any invariant of $G$, the equation $R_{1} I=I$ implies that $I$ is a polynomial in $x_{1}{ }^{{ }}$, whence

$$
x_{1}^{r_{1}-1} \text { divides } \partial I / \partial x_{1} \text {. }
$$

Received July 15, 1959. 
Thus the first row of $J$, and hence also $\operatorname{det} J$, is divisible by

$$
x_{1}^{r_{1}-1} \text {, and hence also by } L_{1}^{r_{1}-1} \text {. }
$$

Similarly, det $J$ is divisible by each $L_{i}^{\tau_{i}-1}$. Using the formula

$$
\sum_{j=1}^{n}\left(d_{j}-1\right)=\sum_{i=1}^{v}\left(r_{i}-1\right),
$$

proved in (4, p. 290,1. 12), a comparison of degrees shows that the factor $c$

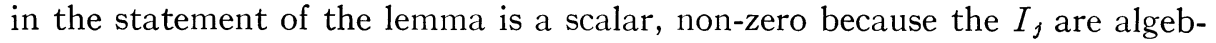
raically independent.

From the first part of the proof we have:

COROLlary. The determinant of the Jacobian of any $n$ invariants of $G$ is divisible by $\prod L_{i}^{r_{i}-1}$.

Proof of the theorem. If $k, l$, and $m$ denote the respective numbers defined by (a), (b), and (c), we prove in turn that $m \leqslant k, k \leqslant l$, and $l \leqslant m$.

First label the $L$ 's so that $L_{1}, \ldots, L_{u}$ are those which contain $p$, and then choose an orthonormal basis $p_{1}, \ldots, p_{n}$ of $V$ so that $p_{1}, \ldots, p_{k}$ span the same subspace as $a_{1}, \ldots, a_{u}$, the normals to the $L$ 's. Let $G^{\prime}$ be the (reflection) group generated by $R_{1}, \ldots, R_{u}$. The co-ordinates $x_{k+1}=I_{k+1}{ }^{\prime}, \ldots, x_{n}=I_{n}^{\prime}$ are invariants of $G^{\prime}$. If $I_{1}{ }^{\prime}, \ldots, I_{k}{ }^{\prime}$ are any invariants of $G$, they are also invariants of $G^{\prime}$, and the corollary above shows that

$$
\prod_{1}^{u} L_{i}^{r_{i}-1}
$$

divides

$$
\partial\left(I_{1}^{\prime}, \ldots, I_{n}^{\prime}\right) / \partial\left(x_{1}, \ldots, x_{n}\right)
$$

that is, divides

$$
\partial\left(I_{1}^{\prime}, \ldots, I_{k}^{\prime}\right) / \partial\left(x_{1}, \ldots, x_{k}\right) .
$$

Consider now the expansion of $\operatorname{det} J$ across the first $k$ rows:

$$
\operatorname{det} J=\sum \pm J^{\prime}\left(i_{1}, \ldots, i_{k}\right) J^{\prime \prime}\left(i_{k+1}, \ldots, i_{n}\right),
$$

with $J^{\prime}\left(i_{1}, \ldots, i_{k}\right)$ denoting the minor corresponding to the rows $1, \ldots, k$ and columns $i_{1}, \ldots, i_{k}$ of $J, J^{\prime \prime}\left(i_{k+1}, \ldots, i_{n}\right)$ denoting the minor corresponding to the rows $k+1, \ldots, n$ and columns $i_{k+1}, \ldots, i_{n}$, and the sum being over all permutations $i_{1}, \ldots, i_{n}$ of $1, \ldots, n$ for which $i_{1}<\ldots<i_{k}$ and $i_{k+1}<\ldots$ $<i_{n}$. By what has just been shown, each $J^{\prime}$ is divisible by

$$
\prod_{1}^{u} L_{i}^{r_{i}-1}
$$

so that, by the lemma, there are polynomials $M\left(i_{1}, \ldots, i_{k}\right)$ such that

$$
\prod_{u+1}^{v} L_{i}^{r_{i-1}}=\sum M\left(i_{1}, \ldots, i_{k}\right) J^{\prime \prime}\left(i_{k+1}, \ldots, i_{n}\right) .
$$


Since the left side of this equation is not 0 at $p$, we conclude that some $J^{\prime \prime}$ is not 0 at $p$, whence $J$ has rank $n-k$ at least and nullity $k$ at most at $p$. Thus $m \leqslant k$.

Next, assume that the labelling is such that $L_{1}, \ldots, L_{k}$ contain $p$ and are linearly independent. Set $x=R_{1} R_{2} \ldots R_{k}$. Suppose $x q=q$, with $q \in V$. Then $R_{1}^{-1} q=R_{2} \ldots R_{k} q$ implies that

$$
q+c_{1} a_{1}=q+c_{2} a_{2}+\ldots+c_{k} a_{k}
$$

for suitable scalars $c_{j}$, whence, because of the linear independence of the $a_{j}$, we conclude that $c_{1}=0$ and $R_{1} q=q$. Similarly $R_{2} q=q, \ldots, R_{k} q=q$, hence $q$ lies in each of $L_{1}, \ldots, L_{k}$, and the solution space of the equation $x q=q$ has dimension $n-k$. Thus $1-x$ has rank $k$, and the inequality $k \leqslant l$ has been established.

Finally choose $x \in G$ so that $1-x$ has rank $l$ and $x p=p$, and then an orthonormal basis $p_{1}, \ldots, p_{n}$ of $V$ so that $x p_{j}=c_{j} p_{j}$ with $c_{j} \neq 1$ for $1 \leqslant j \leqslant l$ and $c_{i}=1$ for $l+1 \leqslant j \leqslant n$. If $I$ is an invariant of $G$, the equation $x I=I$ implies that each term of $I$ has a total exponent in the co-ordinates $x_{1}, \ldots, x_{l}$ which is either 0 or at least 2 . Thus for each $j$ such that $1 \leqslant j \leqslant l, \partial I / \partial x_{j}$ is 0 at any point at which $x_{1}, \ldots, x_{l}$ are all 0 , in particular, at $p$. This implies that the first $l$ rows of $J$ vanish at $p$, whence $l \leqslant m$.

Thus the theorem is completely proved.

\section{References}

1. C. Chevalley, Invariants of finite groups generated by reflections, Amer. J. Math., r7 (1955), 778.

2. H. S. M. Coxeter, The product of the generators of a finite group generated by reflections, Duke Math. J., 18 (1951), 765.

3. G. C. Shephard, Some problems of finite reflection groups, Enseignement Math., $I I(1956), 42$.

4. G. C. Shephard and J. A. Todd, Finite unitary reflection groups, Can. J. Math., 6 (1954), 274.

University of California, Los Angeles 